B. Singh

Nagoya Math. J.

Vol. 103 (1986), 67-84

\title{
DIFFERENTIAL OPERATORS ON A HYPERSURFACE
}

\author{
BALWANT SINGH*
}

\section{Introduction}

We study differential operators on an affine algebraic variety, especially a hypersurface, in the context of Nakai's Conjecture. We work over a field $k$ of characteristic zero. Let $X$ be a reduced affine algebraic variety over $k$ and let $A$ be its coordinate ring. Let $\operatorname{Diff}_{k}^{n}(A)$ be the $A$-module of differential operators of $A$ over $k$ of order $\leqq n$. Nakai's Conjecture asserts that if $\operatorname{Diff}_{k}^{n}(A)$ is generated by $\operatorname{Diff}_{k}^{1}(A)$ for every $n \geqq 2$ then $A$ is regular. In 1973 Mount and Villamayor [6] proved this in the case when $X$ is an irreducible curve. In the general case no progress seems to have been made on the conjecture, except for a result of Brown [2], where the assertion is proved under an additional hypothesis. ${ }^{(1)}$ An interesting result proved by Becker [1] and Rego [8] says that Nakai's Conjecture implies the Conjecture of Zariski-Lipman, which is still open in the general case and which asserts that if the module of $k$-derivations of $A$ is $A$-projective then $A$ is regular.

Write $A=R / J$, where $R$ is a polynomial ring over $k$ and $J$ is an ideal of $R$. Let $\operatorname{Diff}_{k}^{n}(R, A)$ be the $A$-module of differential operators of $R$ into $A$ over $k$ of order $\leqq n$. Since $R$ is a polynomial ring, the structure of $\operatorname{Diff}_{k}^{n}(R, A)$ is well-known, and $\operatorname{Diff}_{k}^{n}(A)$ can be identified with the $A$ submodule of those $D \in \operatorname{Diff}_{k}^{n}(R, A)$ for which $D(J)=0$. In this paper we first analyze the condition " $D(J)=0$ " in some detail, and prove in Proposition (2.10) that for $D(J)$ to be zero it is sufficient (and necessary) that $D$ and certain other differential operators derived from $D$ vanish on a set of generators of $J$. This is then used to prove that if $X$ is a hypersurface, i.e. if we can write $A=R / J$ with $J$ principal, then $\operatorname{Diff}_{k}^{2}(A)$ is completely

Received December 27, 1984.

(*) During the course of this work I was supported generously by the research grant of Leslie Roberts and enjoyed the hospitality of Queen's University. To both of them I express my gratitude.

(1) See additional remarks in the last paragraph of this section. 
determined by $\operatorname{Diff}_{k}^{1}(A)$ (Theorem (2.13)), although, of course, in general, $\operatorname{Diff}_{k}^{2}(A)$ is not generated by $\operatorname{Diff}_{k}^{1}(A)$. This relation between $\operatorname{Diff}_{k}^{1}(A)$ and $\operatorname{Diff}_{k}^{2}(A)$ leads us to consider the following question, which is stronger than Nakai's Conjecture: If $\operatorname{Diff}_{k}^{2}(A)$ is generated by $\operatorname{Diff}_{k}^{1}(A)$ then is $A$ regular? We are able to show that the answer is in the affirmative in the following two cases: (1) if $X$ is a plane curve (Theorem (3.3)); (2) if $X$ is a cone in 3-space (Theorem (5.3)). In the process of proving Theorem (3.3) we get the following interesting result proved in Theorem (3.1): If $X$ is a plane curve then the quotient $\operatorname{Diff}_{k}^{2}(A) / \operatorname{Diff}_{k}^{1}(A) \operatorname{Diff}_{k}^{1}(A)$ is isomorphic to $\mathfrak{a}_{1} \cap \mathfrak{a}_{2} / \mathfrak{a}_{1} \mathfrak{a}_{2}$, where $\mathfrak{a}_{1}, \mathfrak{a}_{2}$ are certain ideals of $A$.

The author would like to express his thanks to Sankar Dutta for supplying a proof of Proposition (3.2).

After this work was submitted for publication, the paper [12] of J.-P. Vigue was brought to our attention by the referee. ${ }^{(1)}$ It follows from Proposition 5 of this paper of Vigue that if $X$ is a normal cone in the complex affine 3 -space and $\operatorname{Diff}_{k}^{2}(A)$ is generated by $\operatorname{Diff}_{k}^{1}(A)$ then $A$ is regular. It has also come to our notice in the meantime that $Y$. Ishibashi has recently proved Nakai's Conjecture in case $X$ is a two-dimensional complete intersection cone (with $k$ algebraically closed) [13] and also in case $X$ is the quotient of an affine $n$-space over $k$ by a finite subgroup of $G L(n, k)$ [14]. There is thus an overlap between our Theorem (5.3) and the results of Vigué and Ishibashi. Our methods, however, are entirely different and more elementary and might therefore be of some interest, since very little seems to be known about the structure of differential operators on $X$.

\section{§1. Preliminaries}

Throughout this paper all rings are assumed to be commutative with 1.

The letter $n$ (resp. $m$ ) will denote either an integer or $\infty$.

Let $k$ be a ring, let $R$ be a $k$-algebra and let $A$ be an $R$-module. The multiplication on $A$ by an element $a \in R$ is denoted $a_{A}$. We shall regard $\operatorname{Hom}_{k}(R, A)$ as an $R$-module via $A$, i.e. for $a \in R$ and $D \in \operatorname{Hom}_{k}(R, A)$ we let $a D=a_{A} \circ D$. For $a \in R$ and $D \in \operatorname{Hom}_{k}(R, A)$ the symbol $[D, a]$ denotes, as usual, the element $D \circ a_{R}-a_{A} \circ D$ of $\operatorname{Hom}_{k}(R, A)$. For $n \in Z$ the $R$ -

(1) The author would like to thank the referee for this and for pointing out some misprints in the manuscript. 
submodule $\operatorname{Diff}_{k}^{n}(R, A)$ of $\operatorname{Hom}_{k}(R, A)$ is defined by induction on $n$ : $\operatorname{Diff}_{k}^{n}(R, A)=0$ if $n<0$, and

$$
\operatorname{Diff}_{k}^{n}(R, A)=\left\{D \in \operatorname{Hom}_{k}(R, A) \mid[D, a] \in \operatorname{Diff}_{k}^{n-1}(R, A) \text { for every } a \in R\right\} .
$$

Elements of $\operatorname{Diff}_{k}^{n}(R, A)$ are called differential operators (of $R$ into $A$ over $k$ ) of order $\leqq n$.

(1.1) Remark. Let $\left\{a_{i}\right\}$ be a set of $k$-algebra generators of $R$. It is easily checked that if $D \in \operatorname{Hom}_{k}(R, A)$ and $\left[D, a_{i}\right] \in \operatorname{Diff}_{k}^{n-1}(R, A)$ for every $i$ then $D \in \operatorname{Diff}_{k}^{n}(R, A)$.

Note that $\operatorname{Diff}_{k}^{0}(R, A)=\operatorname{Hom}_{R}(R, A) \cong A$ and $\operatorname{Diff}_{k}^{n}(R, A) \subset \operatorname{Diff}_{k}^{n+1}(R, A)$ for every $n$. Put $\operatorname{Diff}_{k}^{\infty}(R, A)=\cup_{n \in Z} \operatorname{Diff}_{k}^{n}(R, A)$.

Next, recall from Nakai [7] the definition of a high order derivation. For $n \in Z, n \geqq 0$, an element $D$ of $\operatorname{Hom}_{k}(R, A)$ is called an $n t h$ order derivation (of $R$ into $A$ over $k$ ) if for any $n+1$ elements $a_{0}, \cdots, a_{n}$ of $R$ we have

$$
D\left(a_{0} \cdots a_{n}\right)=\sum_{s=1}^{n}(-1)^{s+1} \sum_{i_{1}<\cdots<i_{s}} a_{i_{1}} \cdots a_{i_{s}} D\left(a_{0} \cdots \hat{a}_{i_{1}} \cdots \hat{a}_{i_{s}} \cdots a_{n}\right),
$$

where $`$ means omitted. Denote by $\operatorname{Der}_{k}^{n}(R, A)$ the $R$-submodule of $\operatorname{Hom}_{k}(R, A)$ consisting of all $n$th order derivations. We have $\operatorname{Der}_{k}^{0}(R, A)=0$ and $\operatorname{Der}_{k}^{1}(R, A)=$ the module of ordinary $k$-derivations of $R$ into $A$. Define $\operatorname{Der}_{k}^{n}(R, A)=0$ if $n<0$.

We note the relation between $\operatorname{Diff}_{k}^{n}(R, A)$ and $\operatorname{Der}_{k}^{n}(R, A)$ in the following lemma. For $x \in A$ let $x_{R}$ denote the $R$-linear map $a \mapsto a x$ of $R$ into $A$.

\section{(1.2) LEMMA.}

(1) Let $D \in \operatorname{Hom}_{k}(R, A)$. Then $D \in \operatorname{Diff}_{k}^{n}(R, A)$ if and only if $D-D(1)_{R}$ $\in \operatorname{Der}_{k}^{n}(R, A)$.

(2) $\operatorname{Der}_{k}^{n}(R, A)=\left\{D \in \operatorname{Diff}_{k}^{n}(R, A) \mid D(1)=0\right\}$. Consequently, $\operatorname{Der}_{k}^{n}(R, A)$ $\subset \operatorname{Der}_{k}^{n+1}(R, A)$ for every $n$.

(3) $\quad$ Put $\operatorname{Der}_{k}^{\infty}(R, A)=\cup_{n \in Z} \operatorname{Der}_{k}^{n}(R, A)$. For $0 \leqq n \leqq \infty$ we have an $R$ linear isomorphism $h$ : $\operatorname{Diff}_{k}^{n}(R, A) \rightarrow A \oplus \operatorname{Der}_{k}^{n}(R, A)$ given by $h(D)=(D(1)$, $\left.D-D(1)_{R}\right)$ and $h^{-1}(x, D)=x_{R}+D$.

Proof. (1) follows from the formula defining an $n$th order derivation and a similar characterization of a differential operator given in [4, (IV, 16.8.8)]. (2) and (3) follow from (1) by noting that if $D \in \operatorname{Der}_{k}^{n}(A)$ then $D(1)=0$. 
Let $S$ be a multiplicatively closed subset of $R$. If $k$ is noetherian and $R$ is a finitely generated $k$-algebra then it follows from [4, (IV, 16.4.15)] that for every $n$ there is a natural isomorphism $S^{-1}\left(\operatorname{Diff}_{k}^{n}(R, A)\right) \cong$ $\operatorname{Diff}_{k}^{n}\left(S^{-1} R, S^{-1} A\right)$. We use this isomorphism to identify these two modules. Then $S^{-1}\left(\operatorname{Der}_{k}^{n}(R, A)\right)=\operatorname{Der}_{k}^{n}\left(S^{-1} R, S^{-1} A\right)$ by Lemma (1.2).

Assume now that $A=R$. Write $\operatorname{Diff}_{k}^{n}(A)$ for $\operatorname{Diff}_{k}^{n}(A, A)$ and $\operatorname{Der}_{k}^{n}(A)$ for $\operatorname{Der}_{k}^{n}(A, A)$. If $D \in \operatorname{Diff}_{k}^{n}(A)$ and $D^{\prime} \in \operatorname{Diff}_{k}^{m}(A)$ then $D D^{\prime} \in \operatorname{Diff}_{k}^{n+m}(A)$ ([4, (IV, 16.8.9)] or [10, Proposition 1]). Further, if $D^{\prime} \in \operatorname{Der}_{k}^{m}(A)$ then $D D^{\prime}(1)=0$, so that $D D^{\prime} \in \operatorname{Der}_{k}^{n+m}(A)$.

For an integer $n \geqq 2$ let us say that $\operatorname{Diff}_{k}^{n}(A)$ is generated by $\operatorname{Diff}_{k}^{1}(A)$ if $\operatorname{Diff}_{k}^{n}(A)$ equals the $A$-submodule of all finite sums of the products $D_{1} \cdots D_{n}$ with $D_{1}, \cdots, D_{n} \in \operatorname{Diff}_{k}^{1}(A)$. We shall say that $\operatorname{Diff}_{k}^{\infty}(A)$ is generated by $\operatorname{Diff}_{k}^{1}(A)$ if $\operatorname{Diff}_{k}^{n}(A)$ is generated by $\operatorname{Diff}_{k}^{1}(A)$ for every $n \geqq 2$.

Denote by $\operatorname{Diff}_{k}^{1}(A) \operatorname{Diff}_{k}^{n-1}(A)$ the $A$-submodule of $\operatorname{Diff}_{k}^{n}(A)$ consisting of all finite sums $\sum_{i} D_{i} D_{i}^{\prime}$ with $D_{i} \in \operatorname{Diff}_{k}^{1}(A), D_{i}^{\prime} \in \operatorname{Diff}_{k}^{n-1}(A)$. Let $\operatorname{Der}_{k}^{1}(A)$ $\operatorname{Der}_{k}^{n-1}(A)$ have a similar meaning.

From Lemma (1.2) we immediately get

(1.3) Lemma. For $n \geqq 2$ we have $\operatorname{Diff}_{k}^{1}(A) \operatorname{Diff}_{k}^{n-1}(A)=\operatorname{Diff}_{k}^{n-1}(A)+$ $\operatorname{Der}_{k}^{1}(A) \operatorname{Der}_{k}^{n-1}(A)$. Moreover the following three conditions are equivalent:

(i ) $\operatorname{Diff}_{k}^{\infty}(A)$ is generated by $\operatorname{Diff}_{k}^{1}(A)$.

(ii) $\operatorname{Diff}_{k}^{n}(A)=\operatorname{Diff}_{k}^{1}(A) \operatorname{Diff}_{k}^{n-1}(A)$ for every $n \geqq 2$.

(iii) $\operatorname{Der}_{k}^{n}(A)=\operatorname{Der}_{k}^{n-1}(A)+\operatorname{Der}_{k}^{1}(A) \operatorname{Der}_{k}^{n-1}(A)$ for every $n \geqq 2$.

Suppose now that $k$ is a field of characteristic zero and $A$ is a finitely generated $k$-algebra. Then it follows from [4, (IV, 16.11.2)] that if $A$ is regular then $\operatorname{Diff}_{k}^{\infty}(A)$ is generated by $\operatorname{Diff}_{k}^{1}(A)$. Nakai's Conjecture asserts the converse:

NAKAI's CoNJECTURE. Let $A$ be a finitely generated algebra over a field $k$ of characteristic zero. If $\operatorname{Diff}_{k}^{\infty}(A)$ is generated by $\operatorname{Diff}_{k}^{1}(A)$ then $A$ is regular.

\section{§. General results}

Let $k$ be a field of characteristic zero. Let $R=k\left[X_{1}, \cdots, X_{r}\right]$ be the polynomial ring in $r$ variables over $k$. Let $J$ be a proper ideal of $R$, let $A=R / J$ and let $\eta: R \rightarrow A$ be the natural map. Put $x_{i}=\eta\left(X_{i}\right)$ for $1 \leqq i$ $\leqq r$.

Let $Z^{+}$be the set of all non-negative integers and put $V=\left(Z^{+}\right)^{r}$. For 
$\alpha=\left(\alpha_{1}, \cdots, \alpha_{r}\right) \in V$ we use the standard notation: $|\alpha|=\alpha_{1}+\cdots+\alpha_{r}$, $\alpha !=\alpha_{1} ! \cdots \alpha_{r} !, X^{\alpha}=X_{1}^{\alpha_{1}} \cdots X_{r}^{\alpha_{r}}$, etc. For $n \in Z$ let $V_{n}=\{\alpha \in V|| \alpha \mid \leqq n\}$ and $W_{n}=\{\alpha \in V|| \alpha \mid=n\}$. For $1 \leqq i \leqq r$ let $e_{i}=(0, \cdots, 1, \cdots, 0) \in W_{1}$ with 1 in the $i$ th place.

For $\alpha \in V$ let $\Delta_{\alpha}: R \rightarrow A$ denote the composite of $(1 / \alpha !) \partial^{\alpha} / \partial X^{\alpha}: R \rightarrow R$ and $\eta$. Then $\Delta_{\alpha} \in \operatorname{Diff}_{k}^{|\alpha|}(R, A)$. It is well-known that every $D \in \operatorname{Diff}_{k}^{\infty}(R, A)$ has a unique expression of the form

$$
D=\sum_{\alpha \in V} c_{\alpha}(D) \Delta_{\alpha}
$$

with $c_{\alpha}(D) \in A$ for all $\alpha$ and $c_{\alpha}(D)=0$ for almost all $\alpha$. Moreover, $D \in$ $\operatorname{Diff}_{k}^{n}(R, A)$ if and only if $c_{\alpha}(D)=0$ for $|\alpha|>n$. On the other hand, $D \in$ $\operatorname{Diff}_{k}^{n}(R, A)$ is also uniquely determined by the values $D\left(X^{\alpha}\right)$ for $\alpha \in V_{n}$.

For $n \in Z$ we define a map $\pi_{n}$ : $\operatorname{Diff}_{k}^{\infty}(R, A) \rightarrow \operatorname{Diff}_{k}^{n}(R, A)$ as follows: For $D \in \operatorname{Diff}_{k}^{\infty}(R, A)$ let $\pi_{n}(D)$ be the unique element of $\operatorname{Diff}_{k}^{n}(R, A)$ determined by $\pi_{n}(D)\left(X^{\alpha}\right)=D\left(X^{\alpha}\right)$ for all $\alpha \in V_{n}$.

In (2.1)-(2.3) below, let $D \in \operatorname{Diff}_{k}^{\infty}(R, A)$, let $\beta \in V$ and let $j \in Z$ with $1 \leqq j \leqq r$.

(2.1) Lemma.

$$
c_{\beta}\left(\left[D, X_{j}\right]\right)=c_{\beta+e_{j}}(D) .
$$

Proof. Immediate from the observation

$$
\left[A_{\beta}, X_{j}\right]= \begin{cases}A_{\beta-e_{j}}, & \text { if } \beta_{j}>0, \\ 0, & \text { if } \beta_{j}=0 .\end{cases}
$$

(2.2) Lemma.

$$
\left[\pi_{n}(D), X_{j}\right]=\pi_{n-1}\left(\left[D, X_{j}\right]\right) .
$$

Proof. Both sides belong to $\operatorname{Diff}_{k}^{n-1}(R, A)$. Therefore it is enough to prove that they coincide on $X^{\alpha}$ for all $\alpha \in V_{n-1}$. Let $\alpha \in V_{n-1}$. Then

$$
\begin{aligned}
{\left[\pi_{n}(D), X_{j}\right]\left(X^{\alpha}\right) } & =\pi_{n}(D)\left(X_{j} X^{\alpha}\right)-X_{j} \pi_{n}(D)\left(X^{\alpha}\right)=D\left(X_{j} X^{\alpha}\right)-X_{j} D\left(X^{\alpha}\right) \\
& =\left[D, X_{j}\right]\left(X^{\alpha}\right)=\pi_{n-1}\left(\left[D, X_{j}\right]\right)\left(X^{\alpha}\right) .
\end{aligned}
$$

(2.3) Proposition.

$$
c_{\beta}(D)=D\left(X^{\beta}\right)-\pi_{|\beta|-1}(D)\left(X^{\beta}\right) .
$$

Proof. Induction on $|\beta|$. Evaluating $D=\sum_{\alpha \in V} c_{\alpha}(D) \Delta_{\alpha}$ on 1 we get $D(1)=c_{0}(D)$, which is our assertion for $|\beta|=0$. Now, let $|\beta|>0$. Choose 
$i$ such that $\beta_{i}>0$, and let $\gamma=\beta-e_{i}$. Then

$$
\begin{aligned}
c_{\beta}(D) & =c_{r}\left(\left[D, X_{i}\right]\right) & & \text { (Lemma (2.1)) } \\
& =\left[D, X_{i}\right]\left(X^{r}\right)-\pi_{|\gamma|-1}\left(\left[D, X_{i}\right]\right)\left(X^{r}\right) & & \text { (Induction) } \\
& =\left[D, X_{i}\right]\left(X^{r}\right)-\left[\pi_{|\beta|-1}(D), X_{i}\right]\left(X^{r}\right) & & \text { (Lemma (2.2)) } \\
& =D\left(X^{\beta}\right)-\pi_{|\beta|-1}(D)\left(X^{\beta}\right), & &
\end{aligned}
$$

since $\pi_{|\beta|-1}(D)\left(X^{r}\right)=D\left(X^{r}\right)$ by definition.

(2.4) Identification. Let us identify

$$
\begin{aligned}
& \operatorname{Diff}_{k}^{n}(A)=\left\{D \in \operatorname{Diff}_{k}^{n}(R, A) \mid D(J)=0\right\} \\
& \operatorname{Der}_{k}^{n}(A)=\left\{D \in \operatorname{Der}_{k}^{n}(R, A) \mid D(J)=0\right\}
\end{aligned}
$$

via the map $D \rightarrow D \eta$.

(2.5) Proposition. Let $D \in \operatorname{Der}_{k}^{1}(A)$ and $D^{\prime} \in \operatorname{Diff}_{k}^{n}(A)$. Then for every $\alpha \in W_{n+1}$ we have

$$
c_{\alpha}\left(D D^{\prime}\right)=\sum_{i=1}^{r} \alpha_{i} D\left(X_{i}\right) c_{\alpha-e_{i}}\left(D^{\prime}\right) .
$$

(Note that if $\alpha_{i}=0$ then $c_{\alpha-e_{i}}\left(D^{\prime}\right)$ is not defined, but then we take the corresponding summand to be zero by standard convention.)

Proof. Induction on $n$. The assertion being clear for $n<0$, let $n \geqq 0$ and let $\alpha \in W_{n+1}$. We may assume that $\alpha=e_{1}+\beta$ with $\beta \in W_{n}$. By induction we have

$$
\begin{aligned}
c_{\beta}\left(D\left[D^{\prime}, X_{1}\right]\right) & =\sum_{i=1}^{r} \beta_{i} D\left(X_{i}\right) c_{\beta-e_{i}}\left(\left[D^{\prime}, X_{1}\right]\right) \\
& =\sum_{i=1}^{r} \beta_{i} D\left(X_{i}\right) c_{\alpha-e_{i}}\left(D^{\prime}\right)
\end{aligned}
$$

by Lemma (2.1). On the other hand, by Lemma (2.1) again we have

$$
c_{\alpha}\left(D D^{\prime}\right)=c_{\beta}\left(\left[D D^{\prime}, X_{1}\right]\right) \text {. }
$$

Thus we need to compare $D\left[D^{\prime}, X_{1}\right]$ and $\left[D D^{\prime}, X_{1}\right]$. Since $D$ is a 1-derivation, we have $\left[D D^{\prime}, X_{1}\right]-D\left[D^{\prime}, X_{1}\right]=D\left(X_{1}\right) D^{\prime}$. Therefore

$$
c_{\beta}\left[D D^{\prime}, X_{1}\right]-c_{\beta}\left(D\left[D^{\prime}, X_{1}\right]\right)=c_{\beta}\left(D\left(X_{1}\right) D^{\prime}\right)=D\left(X_{1}\right) c_{\beta}\left(D^{\prime}\right) \text {. }
$$

Now, by (2.5.1), (2.5.2) and (2.5.3) we have 


$$
\begin{aligned}
c_{\alpha}\left(D D^{\prime}\right) & =c_{\beta}\left(\left[D D^{\prime}, X_{1}\right]\right)=D\left(X_{1}\right) c_{\beta}\left(D^{\prime}\right)+\sum_{i=1}^{r} \beta_{i} D\left(X_{i}\right) c_{\alpha-e_{i}}\left(D^{\prime}\right) \\
& =\sum_{i=1}^{r} \alpha_{i} D\left(X_{i}\right) c_{\alpha-e_{i}}\left(D^{\prime}\right) .
\end{aligned}
$$

(2.6) Definition. For $D \in \operatorname{Diff}_{k}^{\infty}(R, A)$ and $\beta \in V$ define

$$
\left\langle D, X^{\beta}\right\rangle=\sum_{\alpha \in V} c_{\alpha+\beta}(D) \Delta_{\alpha} .
$$

Note that $\langle D, 1\rangle=D$ and if $D \in \operatorname{Diff}_{k}^{n}(R, A)$ then $\left\langle D, X^{\beta}\right\rangle \in \operatorname{Diff}_{k}^{n-|\beta|}(R, A)$.

(2.7) Definition. Let $\Phi: \operatorname{Diff}_{k}^{\infty}(R, A) \times V \rightarrow \operatorname{Der}_{k}^{\infty}(R, A)$ be the pairing defined by $\Phi(D, \beta)=\left\langle D, X^{\beta}\right\rangle-\left(\left\langle D, X^{\beta}\right\rangle(1)\right)_{R}=\left\langle D, X^{\beta}\right\rangle-c_{\beta}(D) \Delta_{0} \quad$ (see Lemma (1.2)). Note that $\Phi$ is $R$-linear in the first variable. Further note that $\Phi$ is the direct limit of the pairings

given by

$$
\Phi_{n, m}: \operatorname{Diff}_{k}^{n}(R, A) \times W_{m} \rightarrow \operatorname{Der}_{k}^{n-m}(R, A)
$$

$$
\Phi_{n, m}(D, \beta)=\left\langle D, X^{\beta}\right\rangle-c_{\beta}(D) \Delta_{0} .
$$

(2.8) Proposition. For $m \leqq n$ we have an exact sequence

$$
0 \rightarrow \operatorname{Diff}_{k}^{m}(R, A) \smile \operatorname{Diff}_{k}^{n}(R, A) \stackrel{\Theta_{n, m}}{\longrightarrow} \underset{\beta \in W_{m}}{\oplus} \operatorname{Der}_{k}^{n-m}(R, A),
$$

where $\Theta_{n, m}(D)=\left(\Phi_{n, m}(D, \beta)\right)_{\beta \in W^{\prime} m}$.

Proof. We have $\Theta_{n, m}\left(\operatorname{Diff}_{k}^{m}(R, A)\right) \subset \operatorname{Der}_{k}^{0}(R, A)=0$. Suppose $\Theta_{n, m}(D)$ $=0$. Then $\left\langle D, X^{\beta}\right\rangle=c_{\beta}(D) \Delta_{0}$ for every $\beta \in W_{m}$. By definition, $\left\langle D, X^{\beta}\right\rangle$ $=\sum_{\alpha \in V} c_{\alpha+\beta}(D) \Delta_{\alpha}$. Therefore by the uniqueness of this expression we have $c_{\alpha+\beta}(D)=0$ for $|\alpha|>0$. This being so for every $\beta \in W_{m}$, we get $c_{\alpha}(D)=0$ for all $\alpha \in V$ with $|\alpha|>m$. This means that $D \in \operatorname{Diff}_{k}^{m}(R, A)$.

(2.9) Lemma. Let $D \in \operatorname{Diff}_{k}^{\infty}(R, A)$, let $\beta, \gamma \in V$ and let $j \in Z$ with $1 \leqq$ $j \leqq r$. Then:

(1) $\left\langle D, X_{j}\right\rangle=\left[D, X_{j}\right]$.

(2) $\left\langle\left\langle D, X^{\beta}\right\rangle, X^{r}\right\rangle=\left\langle D, X^{\beta+r}\right\rangle$.

(3) $\left\langle\left[D, X_{j}\right], X^{\beta}\right\rangle=\left\langle D, X_{j} X^{\beta}\right\rangle$.

(4) If $X^{\beta}=Y_{1} \cdots Y_{s}$ with $Y_{1}, \cdots, Y_{s} \in\left\{X_{1}, \cdots, X_{r}\right\}$ then $\left\langle D, X^{\beta}\right\rangle=$ $\left[\cdots\left[\left[D, Y_{1}\right], Y_{2}\right], \cdots, Y_{s}\right]$.

Proof. (1) follows from Lemma (2.1). For $\alpha \in V$ we have $c_{\alpha}\left(\left\langle\left\langle D, X^{\beta}\right\rangle\right.\right.$, $\left.\left.X^{r}\right\rangle\right)=c_{\alpha+\gamma}\left(\left\langle D, X^{\beta}\right\rangle\right)=c_{\alpha+\gamma+\beta}(D)=c_{\alpha}\left(\left\langle D, X^{\beta+\gamma}\right\rangle\right)$, which proves (2). (3) follows from (1) and (2), and (4) follows from (3). 
(2.10) Proposition. Let $I$ be an ideal of $R$. For $D \in \operatorname{Diff}_{k}^{n}(R, A)$ the following five conditions are equivalent:

(i ) $D(I) \subset I A$.

(ii) $\left[D, X_{i}\right](I) \subset I A$ for all $i, 1 \leqq i \leqq r$, and there exists a set of generators $\left\{f_{j}\right\}$ of $I$ such that $D\left(f_{j}\right) \in I A$ for every $j$.

(iii) $\left\langle D, X^{\beta}\right\rangle(I) \subset I A$ for every $\beta \in V$.

(iv) $\left\langle D, X^{\beta}\right\rangle(I) \subset I A$ for every $\beta \in V_{n-1}$.

(v) There exists a set of generators $\left\{f_{j}\right\}$ of $I$ such that $\left\langle D, X^{\beta}\right\rangle\left(f_{j}\right) \in I A$ for every $\beta \in V_{n-1}$ and for every $j$.

Proof. The implications (i) $\Rightarrow$ (ii) and (iii) $\Rightarrow($ iv) $\Rightarrow(v)$ are trivial, while the implication (i) $\Rightarrow$ (iii) is immediate from part (4) of the above lemma.

(ii) $\Rightarrow$ (i). It is enough to prove that $D\left(X^{\beta} f_{j}\right) \in I A$ for every $\beta \in V$ and for every $j$. For $|\beta|=0$ this is an assumption in (ii). If $|\beta|>0$ then we may assume that $\beta=e_{1}+\gamma$ with $\gamma \in V$. Then $D\left(X^{\beta} f_{j}\right)=\left[D, X_{1}\right]\left(X^{\gamma} f_{j}\right)+$ $X_{1} D\left(X^{\gamma} f_{j}\right)$. Therefore the assertion follows by induction on $|\beta|$.

(v) $\Rightarrow$ (i). Induction on $n$. The assertion being clear for $n \leqq 0$, assume that $n \geqq 1$. We have $\left\langle\left[D, X_{i}\right], X^{\beta}\right\rangle=\left\langle D, X_{i} X^{\beta}\right\rangle$ by Lemma (2.9). Therefore by (v) $\left\langle\left[D, X_{i}\right], X^{\beta}\right\rangle\left(f_{j}\right) \in I A$ for every $\beta \in V_{n-2}$ and for every $j$. So, by induction, $\left[D, X_{i}\right](I) \subset I A$ for every $i, 1 \leqq i \leqq r$. Further, by taking $\beta=0$ in (v) we have $D\left(f_{j}\right) \in I A$ for every $j$. Therefore, by (ii) $\Rightarrow(\mathrm{i}), D(I) \subset I A$.

Applying the proposition with $I=J$ and using the identification (2.4) we get

(2.11) Corollary. For $D \in \operatorname{Diff}_{k}^{n}(R, A)$ the following three conditions are equivalent:

(i ) $D \in \operatorname{Diff}_{k}^{n}(A)$.

(ii) $\left\langle D, X^{\beta}\right\rangle \in \operatorname{Diff}_{k}^{n-|\beta|}(A)$ for every $\beta \in V$.

(iii) $\left\langle D, X^{\beta}\right\rangle \in \operatorname{Diff}_{k}^{n-|\beta|}(A)$ for every $\beta \in V_{n-1}$.

In view of the above corollary the pairings $\Phi_{n, m}$ of Definition (2.7) induce pairings

$$
\varphi_{n, m}: \operatorname{Diff}_{k}^{n}(A) \times W_{m} \longrightarrow \operatorname{Der}_{k}^{n-m}(A) .
$$

It follows from Proposition (2.8) that for $m \leqq n$ we have an exact sequence

$$
0 \rightarrow \operatorname{Diff}_{k}^{m}(A) \smile \operatorname{Diff}_{k}^{n}(A) \stackrel{\theta_{n, m}}{\longrightarrow} \underset{\beta \in W_{m}}{\oplus} \operatorname{Der}_{k}^{n-m}(A)
$$

where $\theta_{n, m}(D)=\left(\varphi_{n, m}(D, \beta)\right)_{\beta \in W_{m}}$. 
(2.12) Definition. For $n \in Z$ define

$$
\begin{aligned}
\mathscr{D}_{k}^{n}(A)= & \left\{\left(d_{\beta}\right)_{\beta \in W_{n-1}} \in \underset{\beta \in W_{n-1}}{\oplus} \operatorname{Der}_{k}^{1}(A) \mid d_{\beta}\left(x_{i}\right)=d_{\gamma}\left(x_{j}\right) \quad\right. \text { whenever } \\
& \left.\beta+e_{i}=\gamma+e_{j}, \beta, \gamma \in W_{n-1}, 1 \leqq i, j \leqq r\right\}
\end{aligned}
$$

If $D \in \operatorname{Diff}_{k}^{n}(A)$ and $\theta_{n, n-1}(D)=\left(d_{\beta}\right)_{\beta \in W_{n-1}}$ then $d_{\beta}\left(x_{i}\right)=c_{\beta+e_{i}}(D)$. It follows that $\operatorname{im}\left(\theta_{n, n-1}\right) \subset \mathscr{D}_{k}^{n}(A)$. Therefore, writing $\theta_{n}=\theta_{n, n-1}$, we have an exact sequence

$$
0 \rightarrow \operatorname{Diff}_{k}^{n-1}(A) \subset \operatorname{Diff}_{k}^{n}(A) \stackrel{\theta_{n}}{\longrightarrow} \mathscr{D}_{k}^{n}(A)
$$

Of particular interest to us is the exact sequence

$$
0 \rightarrow \operatorname{Diff}_{k}^{1}(A) \smile \operatorname{Diff}_{k}^{2}(A) \stackrel{\theta_{2}}{\longrightarrow} \mathscr{D}_{k}^{2}(A)
$$

We note that

$$
\mathscr{D}_{k}^{2}(A)=\left\{\left(d_{1}, \cdots, d_{r}\right) \in \bigoplus_{i=1}^{r} \operatorname{Der}_{k}^{1}(A) \mid d_{i}\left(x_{\jmath}\right)=d_{j}\left(x_{i}\right) \text { for all } i, j\right\}
$$

and that if $D \in \operatorname{Diff}_{k}^{2}(A)$ then $\theta_{2}(D)=\left(d_{1}, \cdots, d_{r}\right)$, where $d_{i} \in \operatorname{Der}_{k}^{1}(A)$ is given by $d_{i}\left(x_{\jmath}\right)=c_{e_{i}+e_{j}}(D)$. In the following theorem we prove that if $J$ is principal then $\theta_{2}$ is surjective.

(2.13) Theorem. Suppose $J$ is principal. Then the sequence

$$
0 \rightarrow \operatorname{Diff}_{k}^{1}(A) \smile \operatorname{Diff}_{k}^{2}(A) \stackrel{\theta_{2}}{\longrightarrow} \mathscr{D}_{k}^{2}(A) \rightarrow 0
$$

is exact.

Proof. We have only to prove the surjectivity of $\theta_{2}$. Let $\left(d_{1}, \cdots, d_{r}\right)$ $\in \mathscr{D}_{k}^{2}(A)$. Let $a_{i j} \in R$ be a lift of $d_{i}\left(x_{j}\right)$ such that $a_{i j}=a_{j i}$ for all $i, j$. Then, since $d_{i}$ is a 1-derivation of $A$ we have

$$
\sum_{j=1}^{r} a_{i j} \partial f / \partial X_{j}=g_{i} f
$$

with $g_{i} \in R$, where $f$ is a generator of $J$. Differentiating $E_{i}$ with respect to $X_{i}$, adding the results over $1 \leqq i \leqq r$, dividing the sum by 2 and remembering that $a_{i j}=a_{j i}$, we get

$$
\sum_{j=1}^{r} b_{j} \partial f / \partial X_{j}+\frac{1}{2} \sum_{j=1}^{r} a_{j j} \partial^{2} f / \partial X_{j}^{2}+\sum_{i<j} a_{i j} \partial^{2} f / \partial X_{i} \partial X_{j}=g f
$$

with $b_{1}, \cdots, b_{r}, g \in R$. Define $D=\sum_{\alpha \in V_{2}} c_{\alpha}(D) \Delta_{\alpha} \in \operatorname{Diff}_{k}^{2}(R, A)$ by $c_{0}(D)=0$, 
$c_{e_{i}}(D)=\eta\left(b_{i}\right)$ and $c_{e_{i}+e_{j}}(D)=\eta\left(a_{i j}\right), 1 \leqq i, j \leqq r$. Then the above equality implies that $D(f)=0$. Moreover, we have $\left\langle D, X_{i}\right\rangle=\sum_{\alpha \in V_{1}} c_{\alpha+e_{i}}(D) \Delta_{\alpha}=$ $\eta\left(b_{i}\right) \Delta_{0}+\sum_{j=1}^{r} \eta\left(a_{i j}\right) \Delta_{e_{j}}$, so that $\left\langle D, X_{i}\right\rangle(f)=\eta\left(b_{i} f+\sum_{j=1}^{r} a_{i j} \partial f / \partial X_{j}\right)=0$ by $\left(E_{i}\right)$. This proves that $\left\langle D, X^{\beta}\right\rangle(f)=0$ for every $\beta \in V_{1}$. Therefore $D(R f)$ $=0$ by Proposition (2.10), and we get $D \in \operatorname{Diff}_{k}^{2}(A)$. Since $c_{e_{i}+e_{j}}(D)=d_{i}\left(x_{j}\right)$, we have $\theta_{2}(D)=\left(d_{1}, \cdots, d_{r}\right)$. This proves that $\theta_{2}$ is surjective.

(2.14) Remark. The above proof actually constructs a $k$-linear right inverse of $\theta_{2}$. For if we define $t\left(d_{1}, \cdots, d_{r}\right)=D$ in the above notation then it is easily checked that $t\left(d_{1}, \cdots, d_{r}\right)$ is independent of the choice of lifts $a_{i j}$ of $d_{i}\left(x_{j}\right)$ with the condition that $a_{i j}=a_{j i}$.

(2.15) Remark. In general, $\theta_{3}$ is not surjective. Example: Take $r=2$ and $J=R f$ with $f=X_{2}^{2}-X_{1}^{3}$. For $\beta \in W_{2}$ define $d_{\beta} \in \operatorname{Der}_{k}^{1}(A)$ as follows: $d_{(2,0)}=8 x_{1} \Delta_{e_{1}}+12 x_{2} \Delta_{e_{2}}, d_{(1,1)}=12 x_{2} \Delta_{e_{1}}+18 x_{1}^{2} \Delta_{e_{2}}, d_{(0,2)}=18 x_{1}^{2} \Delta_{e_{1}}+27 x_{1} x_{2} \Delta_{e_{2}}$. Then $d=\left(d_{(2,0)}, d_{(1,1)}, d_{(0,2)}\right) \in \mathscr{D}_{k}^{3}(A)$. We claim that $d \notin \operatorname{im}\left(\theta_{3}\right)$. For suppose $d=\theta_{3}(D)$ with $D \in \operatorname{Diff}_{k}^{3}(A)$. Put $c_{i j}=c_{(i, j)}(D)$. Then $c_{30}=8 x_{1}, c_{21}=12 x_{2}$, $c_{12}=18 x_{1}^{2}$ and $c_{03}=27 x_{1} x_{2}$. By Corollary (2.11) we have $D(f)=0,\left\langle D, x_{1}\right\rangle(f)$ $=0$ and $\left\langle D, x_{2}\right\rangle(f)=0$. Computing $\left\langle D, x_{2}\right\rangle(f)-2 x_{2} D(f)=0$ we find that $\left(7+6 c_{20}\right) x_{1} x_{2} \in\left(x_{1}^{2}, x_{2}^{2}\right)$. This implies that $7+6 c_{20} \in\left(x_{1}, x_{2}\right)$. From $\left\langle D, x_{1}\right\rangle(f)$ $=0$ we get $\left(6+3 c_{20}\right) x_{1}^{2} \in\left(x_{2}\right)$, which implies that $6+3 c_{20} \in\left(x_{1}, x_{2}\right)$. Thus we get $5 \in\left(x_{1}, x_{2}\right)$, a contradiction.

\section{§3. Differential operators on a plane curve}

With the notation of Section 2, assume that $r=2$ and $J=R f$ is a non-zero, proper, principal ideal of $R$. For $i=1,2$, put $f_{x_{\imath}}=\eta\left(\partial f / \partial X_{i}\right)$ and let $\mathfrak{a}_{i}=\left\{d\left(x_{i}\right) \mid d \in \operatorname{Der}_{k}^{1}(A)\right\}$. Then $\mathfrak{a}_{1}, \mathfrak{a}_{2}$ are ideals of $A$. Recall that in this case we have

$$
\mathscr{D}_{k}^{2}(A)=\left\{\left(d_{1}, d_{2}\right) \in \operatorname{Der}_{k}^{1}(A) \oplus \operatorname{Der}_{k}^{1}(A) \mid d_{1}\left(x_{2}\right)=d_{2}\left(x_{1}\right)\right\} .
$$

Let $\tau: \mathscr{D}_{k}^{2}(A) \rightarrow \mathfrak{a}_{1} \cap \mathfrak{a}_{2}$ be the map defined by $\tau\left(d_{1}, d_{2}\right)=d_{1}\left(x_{2}\right)=d_{2}\left(x_{1}\right)$. Clearly, $\tau$ is $A$-linear and surjective. Put $\sigma=\tau \theta_{2}: \operatorname{Diff}_{k}^{2}(A) \rightarrow \mathfrak{a}_{1} \cap \mathfrak{a}_{2}$.

(3.1) Theorem. Assume that $f_{x_{1}}$ and $f_{x_{2}}$ are non-zero divisors in $A$. Then $\tau$ is an isomorphism, and the sequence

$$
0 \longrightarrow \operatorname{Diff}_{k}^{1}(A) \smile \operatorname{Diff}_{k}^{2}(A) \stackrel{\sigma}{\longrightarrow} a_{1} \cap a_{2} \longrightarrow 0
$$

is exact. Moreover, $\sigma\left(\operatorname{Diff}_{k}^{1}(A) \operatorname{Diff}_{k}^{1}(A)\right)=a_{1} a_{2}$. In particular, we have an A-isomorphism 


\section{$\operatorname{Diff}_{k}^{2}(A) / \operatorname{Diff}_{k}^{1}(A) \operatorname{Diff}_{k}^{1}(A) \cong \mathfrak{a}_{1} \cap \mathfrak{a}_{2} / \mathfrak{a}_{1} \mathfrak{a}_{2}$.}

Proof. If $d \in \operatorname{Der}_{k}^{1}(A)$ then $d\left(x_{1}\right) f_{x_{1}}+d\left(x_{2}\right) f_{x_{2}}=0$. Since $f_{x_{1}}$ and $f_{x_{2}}$ are non-zero divisors, we have $d\left(x_{1}\right)=0$ if and only if $d\left(x_{2}\right)=0$. Consequently, $d=0$ if and only if $d\left(x_{1}\right)=0$ if and only if $d\left(x_{2}\right)=0$. This proves that $\tau$ is an isomorphism. The exactness of the sequence follows now from Theorem (2.13). Since $\operatorname{Diff}_{k}^{1}(A) \operatorname{Diff}_{k}^{1}(A)=\operatorname{Diff}_{k}^{1}(A)+\operatorname{Der}_{k}^{1}(A) \operatorname{Der}_{k}^{1}(A)$ by Lemma (1.3) and $\sigma\left(\operatorname{Diff}_{k}^{1}(A)\right)=0$, the remaining part of the theorem will follow if we show that $\sigma\left(\operatorname{Der}_{k}^{1}(A) \operatorname{Der}_{k}^{1}(A)\right)=a_{1} a_{2}$. Let $D, D^{\prime} \in \operatorname{Der}_{k}^{1}(A)$ and let $\theta_{2}\left(D D^{\prime}\right)=\left(d_{1}, d_{2}\right)$. Then $\sigma\left(D D^{\prime}\right)=\tau\left(d_{1}, d_{2}\right)=d_{1}\left(x_{2}\right)=c_{(1,1)}\left(D D^{\prime}\right)$ by the remarks preceding Theorem (2.13). By Proposition (2.5) we have $c_{(1,1)}\left(D D^{\prime}\right)$ $=D\left(x_{1}\right) c_{(0,1)}\left(D^{\prime}\right)+D\left(x_{2}\right) c_{(1,0)}\left(D^{\prime}\right)=D\left(x_{1}\right) D^{\prime}\left(x_{2}\right)+D\left(x_{2}\right) D^{\prime}\left(x_{1}\right)$. This proves that $\sigma\left(\operatorname{Der}_{k}^{1}(A) \operatorname{Der}_{k}^{1}(A)\right) \subset \mathfrak{a}_{1} a_{2}$. Conversely, let $a_{i} \in \mathfrak{a}_{i}, i=1,2$. Choose $D, D^{\prime} \in$ $\operatorname{Der}_{k}^{1}(A)$ such that $a_{1}=D\left(x_{1}\right), a_{2}=D^{\prime}\left(x_{2}\right)$. Let $b_{1}=D\left(x_{2}\right), b_{2}=D^{\prime}\left(x_{1}\right)$. Then $a_{1} f_{x_{1}}+b_{1} f_{x_{2}}=0$ and $b_{2} f_{x_{1}}+a_{2} f_{x_{2}}=0$. Therefore, since $f_{x_{1}}$ is a non-zero divisor, we get $a_{1} a_{2}=b_{1} b_{2}$. Now, by the above computation we have $\sigma\left(D D^{\prime}\right)$ $=a_{1} a_{2}+b_{1} b_{2}=2 a_{1} a_{2}$. This proves that $a_{1} a_{2} \subset \sigma\left(\operatorname{Der}_{k}^{1}(A) \operatorname{Der}_{k}^{1}(A)\right)$, and the theorem is proved.

(3.2) Proposition. Let $I_{1}, I_{2}$ be ideals of $R$ containing $f$. Assume that $R / I_{1}$ and $R / I_{2}$ are of finite length and that $I_{1}+I_{2} \neq R$. Then $\operatorname{Tor}_{1}^{A}\left(R / I_{1}\right.$, $\left.R / I_{2}\right) \neq 0$.

Proof (S. Dutta). Localizing at a maximal ideal of $R$ containing $I_{1}+I_{2}$, we may assume that $R$ is a regular local ring of dimension two containing $k$. By [3, Chapter XV, Section 5, Case $\mathrm{C}^{\prime}$ and Chapter XVI, Section 5, Case 1] we have an exact sequence

$$
\operatorname{Tor}_{1}^{R}\left(R / I_{1}, A\right) \otimes_{R} R / I_{2} \longrightarrow \operatorname{Tor}_{1}^{R}\left(R / I_{1}, R / I_{2}\right) \longrightarrow \operatorname{Tor}_{1}^{A}\left(R / I_{1}, R / I_{2}\right) \longrightarrow 0 .
$$

Since $f$ is a non-zero divisor in $R$, we have $\operatorname{Tor}_{1}^{R}\left(R / I_{1}, A\right) \cong R / I_{1}$. Therefore the exact sequence becomes

$$
R / I_{1} \otimes_{R} R / I_{2} \longrightarrow \operatorname{Tor}_{1}^{R}\left(R / I_{1}, R / I_{2}\right) \longrightarrow \operatorname{Tor}_{1}^{A}\left(R / I_{1}, R / I_{2}\right) \longrightarrow 0 .
$$

Put $t=$ length $\left(\operatorname{Tor}_{1}^{A}\left(R / I_{1}, R / I_{2}\right)\right)$ and $t_{i}=$ length $\left(\operatorname{Tor}_{i}^{R}\left(R / I_{1}, R / I_{2}\right)\right)$. Thən $t$, $t_{i}$ are non-negative integers and $t_{i}=0$ for $i \neq 0,1,2$. From the exact sequence we have $t-t_{1}+t_{0} \geqq 0$. Since $\operatorname{dim}\left(R / I_{1}\right)+\operatorname{dim}\left(R / I_{2}\right)=0<2$, we have $t_{0}-t_{1}+t_{2}=0$ by [9, Chapter V, Section 3]. Thus wa gat $t \geqq t_{2}$, and it is now enough to prove that $t_{2}>0$. Let 


$$
0 \longrightarrow R^{n} \stackrel{\lambda}{\longrightarrow} R^{n+1} \longrightarrow R \longrightarrow R / I_{2} \longrightarrow 0
$$

be a minimal free resolution of $R / I_{2}$. Let $\mathrm{m}$ be the maximal ideal of $R$. Choose $a \in R$ such that $a \oplus I_{1}$ and $\operatorname{m} a \subset I_{1}$. Let $b=\bar{a} \otimes(1,0, \cdots, 0) \in R / I_{1}$ $\otimes_{R} R^{n}$, where $\bar{a}$ is the natural image of $a$ in $R / I_{1}$. Then $b \neq 0$ and, since $\operatorname{im}(\lambda) \subset \mathfrak{m} R^{n+1}, b \in \operatorname{ker}\left(1_{R / I_{1}} \otimes \lambda\right)=\operatorname{Tor}_{2}^{R}\left(R / I_{1}, R / I_{2}\right)$. This proves that $t_{2}>0$.

(3.3) Theorem. Let $J$ be a proper, principal ideal of $R=k\left[X_{1}, X_{2}\right]$ and let $A=R / J$. Assume that $A$ is reduced.(1) Then $A$ is regular if and only if $\operatorname{Diff}_{k}^{2}(A)=\operatorname{Diff}_{k}^{1}(A) \operatorname{Diff}_{k}^{1}(A)$.

Proof. If $A$ is regular then the equality $\operatorname{Diff}_{k}^{2}(A)=\operatorname{Diff}_{k}^{1}(A) \operatorname{Diff}_{k}^{1}(A)$ follows from [4, (IV, 16.11.2)]. Conversely, suppose $\operatorname{Diff}_{k}^{2}(A)=\operatorname{Diff}_{k}^{1}(A) \operatorname{Diff}_{k}^{1}(A)$. We may assume that $J \neq 0$. Let $J=R f$ and let $f=f_{1} \cdots f_{s}$ be the prime factorization of $f$ in $R$. Since $k$ is infinite, we may make a linear change of variables to assume that $\partial f_{i} / \partial X_{j} \neq 0$ for all $i=1, \cdots, s, j=1,2$. Then, since $f$ is without multiple factors, $f_{x_{1}}$ and $f_{x_{2}}$ are non-zero divisors in $A$. Since $f_{x_{2}} \Delta_{(1,0)}-f_{x_{1}} \Delta_{(0,1)} \in \operatorname{Der}_{k}^{1}(A)$, we have $f_{x_{2}} \in \mathfrak{a}_{1}$ and $f_{x_{1}} \in \mathfrak{a}_{2}$. Therefore $A / \mathfrak{a}_{1}$ and $A / \mathfrak{a}_{2}$ are of finite length. Let $I_{j}$ be the ideal of $R$ containing $f$ such that $\mathfrak{a}_{j}=I_{j} / R f, j=1,2$. Since $\operatorname{Diff}_{k}^{2}(A)=\operatorname{Diff}_{k}^{1}(A) \operatorname{Diff}_{k}^{1}(A)$, we have, by Theorem (3.1), $0=\mathfrak{a}_{1} \cap \mathfrak{a}_{2} / \mathfrak{a}_{1} \mathfrak{a}_{2} \cong \operatorname{Tor}_{1}^{A}\left(A / \mathfrak{a}_{1}, A / \mathfrak{a}_{2}\right)=\operatorname{Tor}_{1}^{A}\left(R / I_{1}, R / I_{2}\right)$. Therefore $I_{1}+I_{2}=R$ by Proposition (3.2). Now, let $\mathfrak{m}$ be a maximal ideal of $A$. Then $\mathfrak{a}_{1}$ or $\mathfrak{a}_{2}$, say $\mathfrak{a}_{1}$, is not contained in $\mathfrak{m}$. This means that there exists $D \in \operatorname{Der}_{k}^{1}(A)$ such that $D\left(x_{1}\right) \oplus \mathfrak{m}$. Since $f_{x_{2}} \neq 0, x_{1}$ is transcendental over $k$. Since $A / \mathfrak{m}$ is algebraic over $k, \mathfrak{m}$ contains a non-zero polynomial $g\left(x_{1}\right) \in k\left[x_{1}\right]$. Choose such $g$ of least degree. Then $D(g)=\eta\left(\partial g / \partial X_{1}\right) D\left(x_{1}\right) \notin \mathfrak{m}$. Thus $D(\mathfrak{m}) \not \subset \mathfrak{m}$. Let $B$ be the $\mathfrak{m} A_{\mathrm{m}}$-adic completion of $A_{\mathrm{m}}$ and let $\mathfrak{n}$ be the maximal ideal of $B$. Then $D$ extends to a 1-derivation $\hat{D}$ of $B$ such that $\hat{D}(\mathfrak{n}) \not \subset \mathfrak{n}$. Therefore by Zariski's Lemma [11, Lemma 4] $B$ is of the form $B=B_{0}[[Y]]$ with $Y$ analytically independent over $B_{0}$. Since $B$, hence $B_{0}$, is reduced and $\operatorname{dim} B_{0}=0, B_{0}$ is regular. Therefore $B$ is regular. This proves that $A_{\mathrm{m}}$ is regular for every maximal ideal $\mathrm{m}$ of $A$.

\section{§4. $A[T]$ and $A\left[T, T^{-1}\right]$}

In this section let $k$ be a noetherian ring, let $A$ be a finitely generated $k$-algebra and let $T$ be an indeterminate over $A$. Let $u: A \hookrightarrow A[T]$ be the natural inclusion. For $i \in Z, i \geqq 0$, let $p_{i}: A[T] \rightarrow A$ be the $A$-linear map defined by $f=\sum_{i \geqq 0}(T-1)^{i} p_{i}(f)$ for $f \in A[T]$. Define $q_{i}: \operatorname{Diff}_{k}^{\infty}(A[T])$

\footnotetext{
(1) See note added in proof.
} 
$\rightarrow \operatorname{Diff}_{k}^{\infty}(A)$ by $q_{i}(D)=p_{i} D u$ for $D \in \operatorname{Diff}_{k}^{\infty}(A[T])$. Note that $p_{i} D u \in \operatorname{Diff}_{k}^{\infty}(A)$, since $p_{i}$ is $A$-linear. Note also that $q_{i}$ maps $\operatorname{Diff}_{k}^{n}(A[T])$ into $\operatorname{Diff}_{k}^{n}(A)$ for every $n$. Further, we have $D u=\sum_{i \geqq 0}(T-1)^{i} u q_{i}(D)$ for $D \in \operatorname{Diff}_{k}^{\infty}(A[T])$.

Regard $\operatorname{Diff}_{k}^{\infty}(A, A[T])$ as an $A[T]$-module in a natural way. For $D \in \operatorname{Diff}_{k}^{\infty}(A, A[T])$ let $\mu(D)$ denote the $k[T]$-linear endomorphism of $A[T]$ obtained from $D$ by extension of scalars, i.e. for $\sum_{i \geqq 0} a_{i} T^{i} \in A[T]$ with $a_{i} \in A, \mu(D)\left(\sum_{i \geqq 0} a_{i} T^{i}\right)=\sum_{i \geqq 0} D\left(a_{i}\right) T^{i}$. Using Remark (1.1) it is easily checked that this gives us an $A[T]$-linear map

$$
\mu: \operatorname{Diff}_{k}^{\infty}(A, A[T]) \longrightarrow \operatorname{Diff}_{k[T]}^{\infty}(A[T]) \subset \operatorname{Diff}_{k}^{\infty}(A[T])
$$

with $\mu\left(\operatorname{Diff}_{k}^{n}(A, A[T])\right) \subset \operatorname{Diff}_{k[T]}^{n}(A[T]) \subset \operatorname{Diff}_{k}^{n}(A[T])$ for every $n$. Identify $\operatorname{Diff}_{k}^{\infty}(A)$ as an $A$-submodule of $\operatorname{Diff}_{k}^{\infty}(A, A[T])$ via $u$, and denote by $\lambda$ the restriction of $\mu$ to $\operatorname{Diff}_{k}^{\infty}(A)$.

(4.1) Lemma. (1) $\sum_{i \geqq 0}(T-1)^{i} u q_{i} \mu=$ identity on $\operatorname{Diff}_{k}^{\infty}(A, A[T])$.

(2) For every non-negative integer $s, q_{0} T^{s} \lambda=i$ dentity on $\operatorname{Diff}_{k}^{\infty}(A)$.

(3) If $D, D^{\prime} \in \operatorname{Diff}_{k}^{\infty}(A)$ then $\lambda\left(D D^{\prime}\right)=\lambda(D) \lambda\left(D^{\prime}\right)$. Consequently, $\lambda\left(\operatorname{Diff}_{k}^{m}(A) \operatorname{Diff}_{k}^{n}(A)\right) \subset \operatorname{Diff}_{k[T]}^{m}(A[T]) \operatorname{Diff}_{k[T]}^{n}(A[T])$ for all $m, n$.

(4) $q_{0}\left(\operatorname{Diff}_{k}^{m}(A[T]) \operatorname{Diff}_{k}^{n}(A[T])\right) \subset \operatorname{Diff}_{k}^{m}(A) \operatorname{Diff}_{k}^{n}(A)$ for all $m, n$.

Proof. (1) and (2) follow from the definition. (3) follows from the fact that each of $\lambda\left(D D^{\prime}\right)$ and $\lambda(D) \lambda\left(D^{\prime}\right)$ is $k[T]$-linear and coincides with $D D^{\prime}$ on $A$. To prove (4), let $D \in \operatorname{Diff}_{k}^{m}(A[T]), D^{\prime} \in \operatorname{Diff}_{k}^{n}(A[T])$. By (1) we have $D^{\prime} u=\sum_{i \geqq 0}(T-1)^{i} u q_{i}\left(\mu\left(D^{\prime} u\right)\right)=\sum_{i \geqq 0}(T-1)^{i} u q_{i}\left(D^{\prime}\right)$. Therefore

and so

$$
D D^{\prime} u=\sum_{i \geqq 0}\left(\left[D,(T-1)^{i}\right]+(T-1)^{i} D\right) u q_{i}\left(D^{\prime}\right)
$$

$$
\begin{aligned}
q_{0}\left(D D^{\prime}\right) & =\sum_{i \geqq 0} p_{0}\left(\left[D,(T-1)^{i}\right]+(T-1)^{i} D\right) u q_{i}\left(D^{\prime}\right) \\
& =\sum_{i \geqq 0} q_{0}\left(\left[D,(T-1)^{i}\right]+(T-1)^{i} D\right) q_{i}\left(D^{\prime}\right)
\end{aligned}
$$

which belongs to $\operatorname{Diff}_{k}^{m}(A) \operatorname{Diff}_{k}^{n}(A)$. This proves (4).

In the following proposition we have identified $\operatorname{Diff}_{k}^{\infty}(A[T])$ as an $A[T]$-submodule of $\operatorname{Diff}_{k}^{\infty}\left(A\left[T, T^{-1}\right]\right)$ via the natural map $\operatorname{Diff}_{k}^{\infty}(A(T]) \rightarrow$ $\left.\operatorname{Diff}_{k}^{\infty}(A[T])\right)_{T}=\operatorname{Diff}_{k}^{\infty}\left(A\left[T, T^{-1}\right]\right)$, which is injective because $T$ is a nonzero divisor in $A[T]$.

(4.2) Proposition. For $D \in \operatorname{Diff}_{k}^{n}(A)$ the following three conditions are equivalent: 
(i ) $\quad D \in \operatorname{Diff}_{k}^{1}(A) \operatorname{Diff}_{k}^{n-1}(A)$.

(ii) $\lambda(D) \in \operatorname{Diff}_{k}^{1}(A[T]) \operatorname{Diff}_{k}^{n-1}(A[T])$.

(iii) $\lambda(D) \in \operatorname{Diff}_{k}^{1}\left(A\left[T, T^{-1}\right]\right) \operatorname{Diff}_{k}^{n-1}\left(A\left[T, T^{-1}\right]\right)$.

Proof. The implication (i) $\Rightarrow$ (ii) follows from Lemma (4.1) (3), while the implication (ii) $\Rightarrow$ (iii) is clear. Now, assume (iii). Then $\lambda(D)=$ $\sum_{j} D_{j} D_{j}^{\prime}$ (finite sum) with $D_{j} \in \operatorname{Diff}_{k}^{1}\left(A\left[T, T^{-1}\right]\right), D_{j}^{\prime} \in \operatorname{Diff}_{k}^{n-1}\left(A\left[T, T^{-1}\right]\right.$ ). Since $A$ is a finitely generated $k$-algebra, there exists a positive integer $s$ such that $T^{s} D_{j} \in \operatorname{Diff}_{k}^{1}(A[T])$ and $T^{s} D_{j}^{\prime} \in \operatorname{Diff}_{k}^{n-1}(A[T])$. Put $\Delta=\sum_{j} T^{s}\left[D_{j}, T^{s}\right] D_{j}^{\prime}$ $\in \operatorname{Diff}_{k}^{n-1}\left(A\left[T, T^{-1}\right]\right)$. We have $\sum_{j}\left(T^{s} D_{j}\right)\left(T^{s} D_{j}^{\prime}\right)=\Delta+T^{2 s} \lambda(D)$. Therefore $\Delta \in \operatorname{Diff}_{k}^{n-1}(A[T])$. Now, $q_{0}\left(\sum_{j}\left(T^{s} D_{j}\right)\left(T^{s} D_{j}^{\prime}\right)\right) \in \operatorname{Diff}_{k}^{1}(A) \operatorname{Diff}_{k}^{n-1}(A)$ by Lemma (4.1) (4), and $q_{0}(\Delta) \in \operatorname{Diff}_{k}^{n-1}(A) \subset \operatorname{Diff}_{k}^{1}(A) \operatorname{Diff}_{k}^{n-1}(A)$. Therefore $q_{0}\left(T^{2 s} \lambda(D)\right)$ $\in \operatorname{Diff}_{k}^{1}(A) \operatorname{Diff}_{k}^{n-1}(A)$, and now (i) follows by Lemma (4.1) (2).

(4.3) Corollary. If $\operatorname{Diff}_{k}^{n}(A[T])=\operatorname{Diff}_{k}^{1}(A[T]) \operatorname{Diff}_{k}^{n-1}(A[T])$ or $\operatorname{Diff}_{k}^{n}\left(A\left[T, T^{-1}\right]\right)=\operatorname{Diff}_{k}^{1}\left(A\left[T, T^{-1}\right]\right) \operatorname{Diff}_{k}^{n-1}\left(A\left[T, T^{-1}\right]\right)$ then $\operatorname{Diff}_{k}^{n}(A)=$ $\operatorname{Diff}_{k}^{1}(A) \operatorname{Diff}_{k}^{n-1}(A)$.

\section{§5. Differential operators on a cone in 3-space}

Let us return now to the notation of Section 2. For $\alpha \in V$ and $g \in R$, put $g_{x^{\alpha}}=\eta\left(\partial^{\alpha} g / \partial X^{\alpha}\right)$.

(5.1) Lemma. Assume that $J=R f$ and that $x_{1}, f_{x_{r}}$ is an A-regular sequence. Let $D \in \operatorname{Diff}_{k}^{n}(A)$. Suppose $c_{\alpha}(D) \in x_{1} A$ for all those $\alpha \in V_{n}$ for which $\alpha_{r}=0$. Then there exists $D^{\prime} \in \operatorname{Diff}_{k}^{n}(A)$ such that $D=x_{1} D^{\prime}$.

Proof. Since $x_{1}$ is a non-zero divisor in $A$, it is enough to prove that $c_{\alpha}(D) \in x_{1} A$ for every $\alpha \in V_{n}$. We do this by lexicographic induction on $\left(n-|\alpha|, \alpha_{r}\right)$. Let $\alpha \in V_{n}$. The result being given for $\alpha_{r}=0$, we may assume that $\alpha_{r} \geqq 1$. Put $\beta=\alpha-e_{r}$. By Corollary (2.11) $\left\langle D, X^{\beta}\right\rangle \in \operatorname{Diff}_{k}^{n-|\beta|}(A)$. Therefore, since $c_{\beta}(D) \Delta_{0}(f)=0$, we have

$$
0=\left\langle D, X^{\beta}\right\rangle(f)=\sum_{\substack{r \in V_{n-1 \beta}|r|>0 \\|\gamma| \beta}} c_{\gamma+\beta}(D) \Delta_{\gamma}(f) .
$$

Let $\gamma \in V_{n-|\beta|}$ with $|\gamma|>0$ and $|\gamma+\beta| \leqq|\alpha|$. Then $|\gamma|=1,|\gamma+\beta|=|\alpha|$ and either $\gamma=e_{r}$ or $\gamma_{r}=0$. It follows that for every index $\gamma \neq e_{r}$ appearing in the above summation we have $\left(n-|\gamma+\beta|, \gamma_{r}+\beta_{r}\right)<\left(n-|\alpha|, \alpha_{r}\right)$ lexicographically. Therefore by induction we get $c_{\alpha}(D) \Delta_{e_{r}}(f) \in x_{1} A$. Since $\Delta_{e_{r}}(f)=f_{x_{r}}$ and $x_{1}, f_{x_{r}}$ is $A$-regular, we get $c_{\alpha}(D) \in x_{1} A$. 
(5.2) Lemma. Let $H \in k[Y, Z]$ be a homogeneous polynomial in two variables $Y, Z$ over $k$, of degree $\geqq 2$, monic in $Z$ and without multiple factors. Then $(\partial H / \partial Z)^{2}$ does not belong to the ideal $\left(Y^{2} \partial H / \partial Y, Y^{2} \partial H / \partial Z, H\right)$ of $k[Y, Z]$.

Proof. Replacing $k$ by its algebraic closure, we may assume that $H=\prod_{i=1}^{n}\left(Z-t_{i} Y\right)$ with $n=\operatorname{deg}(H)$ and $t_{1}, \cdots, t_{n}$ distinct elements of $k$. Suppose $(\partial H / \partial Z)^{2}=F Y^{2} \partial H / \partial Y+G Y^{2} \partial H / \partial Z+K H$ with $F, G, K \in k[Y, Z]$, $F, G$ homogeneous of degree $n-3$, and $K$ homogeneous of degree $n-2$. Putting $Y=1$ and $Z=t_{i}$ in this identity for a fixed $i$ and dividing the result by $\prod_{j \neq i}\left(t_{i}-t_{j}\right)$ we get $\prod_{j \neq i}\left(t_{i}-t_{j}\right)=g_{0}+\left(g_{1}-f_{0}\right) t_{i}+\cdots+\left(g_{n-3}\right.$ $\left.-f_{n-4}\right) t_{i}^{n-3}-f_{n-3} t_{i}^{n-2}$ for $1 \leqq i \leqq n$, where $f_{j}, g_{j} \in k$ are defined by $F=$ $\sum_{j=0}^{n-3} f_{j} Y^{n-3-j} Z^{j}, G=\sum_{j=0}^{n-3} g_{j} Y^{n-3-j} Z^{j}$. It follows that if we define $C=\left(c_{i j}\right)_{1 \leqq i, j \leqq n}$ with $c_{i 1}=\prod_{j \neq i}\left(t_{i}-t_{j}\right)$ and $c_{i j}=t_{i}^{j-2}$ for $j \geqq 2$ then $\operatorname{det}(C)=0$. On the other hand, it is easily checked that, since $t_{1}, \cdots, t_{n}$ are distinct, $\operatorname{det}(C) \neq 0$. This contradiction proves the lemma.

(5.3) Theorem. Let $J$ be a homogeneous, proper, pricipal ideal of $R=k\left[X_{1}, X_{2}, X_{3}\right]$ and let $A=R / J$. Assume that $A$ is reduced..$^{(1)}$ Then $A$ is regular if and only if $\operatorname{Diff}_{k}^{2}(A)=\operatorname{Diff}_{k}^{1}(A) \operatorname{Diff}_{k}^{1}(A)$.

Proof. If $A$ is regular then the equality $\operatorname{Diff}_{k}^{2}(A)=\operatorname{Diff}_{k}^{1}(A) \operatorname{Diff}_{k}^{1}(A)$ follows from $[4,(\mathrm{IV}, 16.11 .2)]$. Conversely, suppose $\operatorname{Diff}_{k}^{2}(A)=\operatorname{Diff}_{k}^{1}(A)$ - $\operatorname{Diff}_{k}^{1}(A)$. Let $T$ be a homogeneous element of $A$ of degree one. Then $\operatorname{Diff}_{k}^{2}\left(A_{T}\right)=\operatorname{Diff}_{k}^{1}\left(A_{T}\right) \operatorname{Diff}_{k}^{1}\left(A_{T}\right)$. Since $A_{T}=A_{(T)}\left[T, T^{-1}\right]$ with $T$ algebraically independent over $A_{(T)}$, it follows from Corollary (4.3) that

$$
\operatorname{Diff}_{k}^{2}\left(A_{(T)}\right)=\operatorname{Diff}_{k}^{1}\left(A_{(T)}\right) \operatorname{Diff}_{k}^{1}\left(A_{(T)}\right)
$$

Now, since $\operatorname{deg}(T)=1, A_{(T)}$ is a reduced ring of the form $k\left[Y_{1}, Y_{2}\right] /\left(f\left(Y_{1}\right.\right.$, $\left.Y_{2}\right)$ ). Therefore by (5.3.1) and Theorem (3.3) $A_{(T)}$ is regular. This proves that the curve Proj $(A)$ is non-singular. Therefore the only possible singularity of the cone $\operatorname{Spec}(A)$ is its vertex. Also, $A$ is Cohen-Macaulay. Therefore $A$ is normal.

Suppose $A$ is not regular. Let $J=R F$ with $F=F\left(X_{1}, X_{2}, X_{3}\right)$ homogeneous, and let $n=\operatorname{deg}(F)$. Then $n \geqq 2$. Since $\operatorname{char}(k)=0$, we can arrange the following by a homogeneous change of variables: First, $F=X_{3}^{n}+\sum_{j=1}^{n} F_{j} X_{3}^{n-j}$ with $F_{j} \in k\left[X_{1}, X_{2}\right]$ homogeneous of degree $j$ for every

\footnotetext{
(1) See note added in proof.
} 
$j$, and $F_{1}=0$. Then, since $A$ is reduced, $\operatorname{Disc}_{X_{3}}(F)$, the discriminant of $F$ with respect to $X_{3}$, is non-zero. We choose $X_{1}$ such that $\operatorname{Disc}_{X_{3}}(F) \notin$ $X_{1} k\left[X_{1}, X_{2}\right]$. Then $\operatorname{Disc}_{X_{3}}\left(F\left(0, X_{2}, X_{3}\right)\right) \neq 0$ and it follows that $F\left(0, X_{2}, X_{3}\right)$ and $\partial F\left(0, X_{2}, X_{3}\right) / \partial X_{3}$ have no common factors, and $F\left(0, X_{2}, X_{3}\right)$ has no multiple factors.

Having arranged the above, we claim the following:

(1) $\partial F / \partial X_{2} \in\left(X_{1}, X_{2}\right)$.

(2) $\left(\partial F / \partial X_{3}\right)^{2} \notin\left(X_{1}, X_{2}^{2} \partial F / \partial X_{2}, X_{2}^{2} \partial F / \partial X_{3}, F\right)$.

(3) $F, X_{1}, \partial F / \partial X_{3}$ is an $R$-regular sequence.

(4) $\left(\partial F / \partial X_{3}\right)^{2} \in(F)+\left(X_{1}, X_{2}\right)^{2}$.

Note that (1) and (4) follow from the fact that $F_{1}=0$. (2) follows from Lemma (5.2) applied to $H=F\left(0, X_{2}, X_{3}\right)$. To prove (3), first note that $X_{1}$, $F, \partial F / \partial X_{3}$ is an $R$-regular sequence by our choice of variables. Then, since these three elements are homogeneous, any permutation of these is again $R$-regular. This proves our claim.

Now, given $D \in \operatorname{Diff}_{k}^{\infty}(R, A)$, put $\varepsilon(D)=\sum_{\alpha \in V, \alpha_{3}=0} c_{\alpha}(D) \Delta_{\alpha}$. In particular, if $D \in \operatorname{Diff}_{k}^{\infty}(A)$ than we have $\varepsilon(D) \in \operatorname{Diff}_{k}^{\infty}(R, A)$ (see (2.4)).

Since $A$ is normal, it follows from [5, p. 893] that $\operatorname{Der}_{k}^{1}(A)$ is generated as an $A$-module by $d, d_{1}, d_{2}, d_{3}$ where $d=x_{1} \Delta_{e_{1}}+x_{2} \Delta_{e_{2}}+x_{3} \Delta_{e_{3}}, d_{1}=F_{x_{3}} \Delta_{e_{2}}$ $-F_{x_{2}} \Delta_{e_{3}}, d_{2}=F_{x_{3}} \Delta_{e_{1}}-F_{x_{1}} \Delta_{e_{3}}$ and $d_{3}=F_{x_{2}} \Delta_{e_{1}}-F_{x_{1}} \Delta_{e_{2}}$. Consider the elements $d^{2}, d_{1} d, d_{1}^{2}$ of $\operatorname{Diff}_{k}^{2}(A)$. Using Proposition (2.5) it is easily checked that we have

$$
\begin{aligned}
& \varepsilon\left(d^{2}\right)=x_{1} \Delta_{e_{1}}+x_{2} \Delta_{e_{2}}+2 x_{1}^{2} \Delta_{2 e_{1}}+2 x_{1} x_{2} \Delta_{e_{1}+e_{2}}+2 x_{2}^{2} \Delta_{2 e_{2}}, \\
& \varepsilon\left(d_{1} d\right)=F_{x_{3}} \Delta_{e_{2}}+x_{1} F_{x_{3}} \Delta_{e_{1}+e_{2}}+2 x_{2} F_{x_{3}} \Delta_{2 e_{2}}, \\
& \varepsilon\left(d_{1}^{2}\right)=a \Delta_{e_{2}}+2\left(F_{x_{3}}\right)^{2} \Delta_{2 e_{2}},
\end{aligned}
$$

where $a=F_{x_{2} x_{3}} F_{x_{3}}-F_{x_{3}^{2}} F_{x_{2}}$. By (4) we can write $\left(F_{x_{3}}\right)^{2}=b_{1} x_{1}+b_{2} x_{2}^{2}$ with $b_{1}, b_{2} \in A$ homogeneous and $\operatorname{deg}\left(b_{2}\right)=2 n-4$. By (1) we can write $a=$ $u F_{x_{3}}+v_{1} x_{1}+v_{2} x_{2}$ with $u, v_{1}, v_{2} \in A$. Put $D=\left(b_{2}-v_{2}\right) d-u d_{1}-b_{2} d^{2}+d_{1}^{2}$ $\in \operatorname{Diff}_{k}^{2}(A)$. Then an easy computation shows that

$$
\varepsilon(D)=-v_{2} x_{1} \Delta_{e_{1}}+v_{1} x_{1} \Delta_{e_{2}}-2 x_{1}^{2} b_{2} \Delta_{2 e_{1}}-2 x_{1} x_{2} b_{2} \Delta_{e_{1}+e_{2}}+2 x_{1} b_{1} \Delta_{2 e_{2}} .
$$

Thus $c_{\alpha}(D) \in x_{1} A$ for all those $\alpha \in V_{2}$ for which $\alpha_{3}=0$. Therefore it follows from (3) and Lemma (5.1) that there exists $D^{\prime} \in \operatorname{Diff}_{k}^{2}(A)$ such that $D=x_{1} D^{\prime}$. We have $c_{2 e_{1}}\left(D^{\prime}\right)=x_{1}^{-1} c_{2 e_{1}}(D)=-2 x_{1} b_{2}$. Let

$$
\mathfrak{a}=\left\{y \in A \mid \exists \delta \in \operatorname{Diff}_{k}^{2}(A) \text { with } c_{2 e_{\mathbf{1}}}(\delta)=y\right\} .
$$


Then $\mathfrak{a}$ is an ideal of $A$ and $x_{1} b_{2} \in \mathfrak{a}$. Now, we have $\operatorname{Diff}_{k}^{2}(A)=\operatorname{Diff}_{k}^{1}(A)$ - $\operatorname{Diff}_{k}^{1}(A)$ by assumption. So $\operatorname{Diff}_{k}^{2}(A)=\operatorname{Diff}_{k}^{1}(A)+\operatorname{Der}_{k}^{1}(A) \operatorname{Der}_{k}^{1}(A)$ by Lemma (1.3). Therefore, since $\operatorname{Der}_{k}^{1}(A)$ is generated by $d, d_{1}, d_{2}, d_{3}$, it follows from Proposition (2.5) that $a$ is generated by $x_{1}^{2}, x_{1} F_{x_{2}}, x_{1} F_{x_{3}},\left(F_{x_{2}}\right)^{2}$, $F_{x_{2}} F_{x_{3}},\left(F_{x_{3}}\right)^{2}$. All these elements are homogeneous and $\operatorname{deg}\left(\left(F_{x_{2}}\right)^{2}\right)=$ $\operatorname{deg}\left(F_{x_{2}} F_{x_{3}}\right)=\operatorname{deg}\left(\left(F_{x_{3}}\right)^{2}\right)=2 n-2>2 n-3=\operatorname{deg}\left(x_{1} b_{2}\right)$. It follows that $x_{1} b_{2} \in$ $\left(x_{1}^{2}, x_{1} F_{x_{2}}, x_{1} F_{x_{3}}\right)$. Therefore, since $x_{1}$ is a non-zero divisor, $b_{2} \in\left(x_{1}, F_{x_{2}}, F_{x_{3}}\right)$. Since $\left(F_{x_{3}}\right)^{2}=b_{1} x_{1}+b_{2} x_{2}^{2}$, we get $\left(F_{x_{3}}\right)^{2} \in\left(x_{1}, x_{2}^{2} F_{x_{2}}, x_{2}^{2} F_{x_{3}}\right)$. This contradicts (2), and the theorem is proved.

\section{§6. A remark on the non-reduced case}

In proving our results in Sections 3 and 5 we have assumed that $A$ is reduced. We believe that this condition can be dropped. ${ }^{(1)}$ Let us show this in the case $\operatorname{dim}(A)=0$. With the notation of Section 2 let $r=1$, and omit the subscript 1 . Assume that $J=R f$ is non-zero, proper and principal. Then $A=k[x]=k[X] /(f)$ and $\Delta=\eta \partial / \partial X$. Put $f_{x}=\eta(\partial f / \partial X)$.

(6.1) Proposition. If $\operatorname{Diff}_{k}^{2}(A)=\operatorname{Diff}_{k}^{1}(A) \operatorname{Diff}_{k}^{1}(A)$ then $A$ is reduced, hence regular.

Proof. Let

$$
0 \rightarrow \operatorname{Diff}_{k}^{1}(A) \subset \operatorname{Diff}_{k}^{2}(A) \stackrel{\theta_{2}}{\longrightarrow} \operatorname{Der}_{k}^{1}(A) \longrightarrow 0
$$

be the exact sequence of Theorem (2.13). Recall that in this case $\theta_{2}(D)$ $=c_{2}(D) \Delta$. Identify $\operatorname{Der}_{k}^{1}(A)$ with the ideal $\mathfrak{a}=\left\{d(x) \mid d \in \operatorname{Der}_{k}^{1}(A)\right\}$ of $A$ by sending $d \in \operatorname{Der}_{k}^{1}(A)$ to $d(x)$. Then it follows from Proposition (2.5) that $\theta_{2}\left(\operatorname{Der}_{k}^{1}(A) \operatorname{Der}_{k}^{1}(A)\right)=a^{2}$. By our assumption and Lemma (1.3) we have $\operatorname{Diff}_{k}^{2}(A)=\operatorname{Diff}_{k}^{1}(A)+\operatorname{Der}_{k}^{1}(A) \operatorname{Der}_{k}^{1}(A)$. Therefore $\mathfrak{a}=\mathfrak{a}^{2}$. Now, $\mathfrak{a}=\{a \in$ $\left.A \mid a f_{x}=0\right\}$. It follows that if $f=f_{1}^{t_{1}} \cdots f_{m}^{t_{m}}$ is the prime factorization of $f$ with $f_{1}, \cdots, f_{m}$ mutually coprime then $\mathfrak{a}=\left(\eta\left(f_{1} \cdots f_{m}\right)\right)$. Thus the equality $\mathfrak{a}=\mathfrak{a}^{2}$ implies that $f_{1} \cdots f_{m}$ belongs to the ideal $\left(f, f_{1}^{2} \cdots f_{m}^{2}\right)$ of $R$. This is possible only if $t_{1}=\cdots=t_{m}=1$, i.e., if $A$ is reduced.

Note ADDED IN PROOF. It was recently proved by the author jointly with D. P. Patil that if $X$ is a hypersurface and $\operatorname{Diff}_{k}^{2}(A)=\operatorname{Diff}_{k}^{1}(A) \operatorname{Diff}_{k}^{1}(A)$ then $A$ is reduced. Thus, in Theorems (3.3) and (5.3) we can drop the assumption that $A$ be reduced.

(1) See note added in proof. 


\section{REFERENCES}

[1] J. Becker, Higher derivations and integral closure, Amer. J. Math., 100 (1978), 495-521.

[2] W. C. Brown, Higher derivations on finitely generated integral domains, Proc. Amer. Math. Soc., 42 (1974), 23-27.

[ 3 ] H. Cartan and S. Eilenberg, Homological Algebra, Princeton Univ. Press, Princeton 1956.

[ 4 ] A. Grothendieck, Éléments de Géométrie Algébrique, Publ. Math. IHES, 32 (1967).

[5] J. Lipman, Free derivation modules on algebraic varieties, Amer. J. Math., 87 (1965), 874-898.

[6] K. R. Mount and O. E. Villamayor, On a conjecture of Y. Nakai, Osaka J. Math., 10 (1973), 325-327.

[ 7 ] Y. Nakai, High order derivations I, Osaka J. Math., 7 (1970), 1-27.

[ 8 ] C. J. Rego, Remarks on differential operators on algebraic varieties, Osaka J. Math., 14 (1977), 481-486.

[ 9 ] J.-P. Serre, Algèbre locale-Multiplicités, Springer-Verlag Lecture Notes in Math., No. 11, Berlin-Heidelberg-New York, 1975.

[10] J.-P. Vigué, Opérateurs différentiels sur les espaces analytiques, Invent. math., 20 (1973), 313-336.

[11] O. Zariski, Studies in equisingularity I, Amer. J. Math., 87 (1965), 507-536.

[12] J.-P. Vigué, Opérateurs différentiels sur les cônes normaux de dimension 2, C. R. Acad. Sc. Paris, 278, Série A, (1974), 1047-1050.

[13] Y. Ishibashi, Remarks on a Conjecture of Nakai, J. Algebra, 95 (1985), 31-45.

[14] — Nakai's conjecture for invariant subrings, Hiroshima Math. J., 15 (1985), $429-436$.

Tata Institute of Fundamental Research

Homi Bhabha Road,

Bombay 400005 ,

India 